\title{
Regional variations of mesospheric gravity-wave momentum flux over Antarctica
}

\author{
P. J. Espy ${ }^{1}$, R. E. Hibbins ${ }^{1}$, G. R. Swenson ${ }^{2}$, J. Tang ${ }^{2}$, M. J. Taylor ${ }^{3}$, D. M. Riggin ${ }^{4}$, and D. C. Fritts ${ }^{4}$ \\ ${ }^{1}$ The British Antarctic Survey, NERC, Cambridge, UK \\ ${ }^{2}$ Department of Electrical \& Computer Engineering, University of Illinois at Urbana-Champaign, USA \\ ${ }^{3}$ Department of Physics, Utah State University, Logan, Utah, USA \\ ${ }^{4}$ Colorado Research Associates, a division of NorthWest Research Associates, Boulder, Colorado, USA
}

Received: 6 June 2005 - Revised: 2 December 2005 - Accepted: 21 December 2005 - Published: 7 March 2006

\begin{abstract}
Images of mesospheric airglow and radar-wind measurements have been combined to estimate the difference in the vertical flux of horizontal momentum carried by highfrequency gravity waves over two dissimilar Antarctic stations. Rothera $\left(67^{\circ} \mathrm{S}, 68^{\circ} \mathrm{W}\right)$ is situated in the mountains of the Peninsula near the edge of the wintertime polar vortex. In contrast, Halley $\left(76^{\circ} \mathrm{S}, 27^{\circ} \mathrm{W}\right)$, some $1658 \mathrm{~km}$ to the southeast, is located on an ice sheet at the edge of the Antarctic Plateau and deep within the polar vortex during winter. The cross-correlation coefficients between the vertical and horizontal wind perturbations were calculated from sodium (Na) airglow imager data collected during the austral winter seasons of 2002 and 2003 at Rothera for comparison with the 2000 and 2001 results from Halley reported previously (Espy et al., 2004). These cross-correlation coefficients were combined with wind-velocity variances from coincident radar measurements to estimate the daily averaged upper-limit of the vertical flux of horizontal momentum due to gravity waves near the peak emission altitude of the $\mathrm{Na}$ nightglow layer, $90 \mathrm{~km}$. The resulting momentum flux at both stations displayed a large day-to-day variability and showed a marked seasonal rotation from the northwest to the southwest throughout the winter. However, the magnitude of the flux at Rothera was about 4 times larger than that at Halley, suggesting that the differences in the gravity-wave source functions and filtering by the underlying winds at the two stations create significant regional differences in wave forcing on the scale of the station separation.
\end{abstract}

Keywords. Meteorology and atmospheric dynamics (General circulation; Middle atmosphere dynamics; Waves and tides)

\section{Introduction}

Atmospheric gravity waves, with spatial scales of ten to a few hundred kilometers and temporal scales between five minutes and several hours, are typically generated in the lower

Correspondence to: P. J. Espy

(pje@bas.ac.uk) atmosphere through the action of weather systems or orographic lifting of air masses (Fritts and Alexander, 2003, and references therein). These waves grow in amplitude as they propagate into the rarified mesosphere, where some break and dissipate their energy and momentum locally. Depending upon the filtering action of the winds below, this input of momentum closes the mesospheric jets, limiting wind speeds and driving the mesopause winds and temperatures far from radiative equilibrium (Haurwitz, 1961; Garcia and Solomon, 1985; Lübken et al., 1990; Fritts and Luo, 1995; Luo et al., 1995; Espy and Stegman, 2002; Fritts and Alexander, 2003).

Given the role of gravity waves in determining global circulation and thermal balance, they are an essential element of global circulation models (GCMs). However, most of the gravity-wave spectrum that is important for maintaining the mean circulation is not explicitly resolvable in current GCMs (Vincent, 1984), and consequently, the effects of these waves must be parameterized a priori (Hamilton, 1996). More importantly, GCMs require spatial and temporal variations in the gravity-wave parameterization in order to reproduce the wave-driven circulation effects (e.g. Rind et al., 1988; Hamilton, 1995), particularly in the Antarctic where these variations are critical for GCM performance but where measurements are scarce (Garcia and Boville, 1994; Hamilton, 1996). Hence, knowledge of the seasonal and latitudinal behaviour of the gravity-wave momentum flux is essential to both guide and constrain these parameterizations.

In this paper, we present optical-imaging $\mathrm{Na}$ airglow measurements that provide an independent measure of the crosscorrelation between the vertical and horizontal wind perturbations caused by gravity waves in the mesosphere. Combining these with wind-velocity variances from coincident radar measurements, the daily averaged upper-limit of the vertical flux of horizontal momentum due to gravity waves may be inferred. The measurements were undertaken at Rothera for comparison with the climatology of momentum flux reported from Halley (Espy et al., 2004). The two Antarctic stations are separated by $8^{\circ}$ of latitude, and each is subject to extremely different wind regimes and orography. Halley is located on the Brunt Ice Shelf at the edge of the Antarctic plateau and separated from the Trans-Antarctic Mountains 

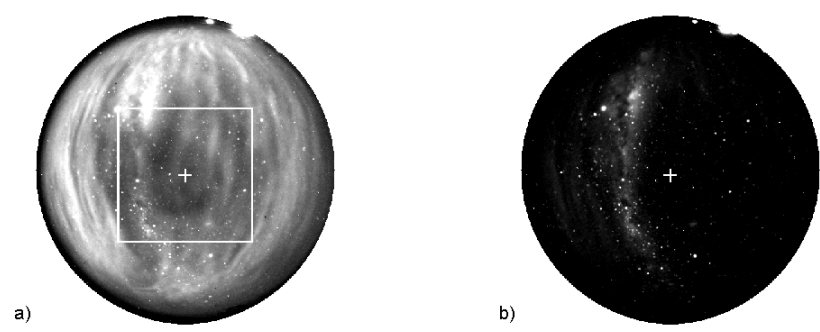

Fig. 1. An all-sky image in sodium emission (a), and at a background wavelength (b), shown on the same gray-scale range. North is at the top and East to the right of the images, and the approximate $150 \mathrm{~km}$ field-of- view analyzed is shown on the Na image. Gravity-wave structures aligned N-S, and moving towards the west, are apparent in the $\mathrm{Na}$ airglow image, whilst the stellar and galactic backgrounds that must be removed before processing are more obvious in the background image.

by $\sim 40^{\circ}$ of longitude. In addition, at $76^{\circ} \mathrm{S}$, it is located deep within the polar vortex during winter. In contrast, Rothera is situated within in the mountains of the Antarctic Peninsula, and at $67^{\circ} \mathrm{S}$ sits near the edge of the strong wintertime polar vortex winds. Thus, the two stations are likely to have differences in their gravity-wave fields. For example, Ern et al. (2004), using $\sim 2$ weeks of satellite temperature data, observed high values of gravity-wave momentum flux at $25 \mathrm{~km}$ at the point where the Peninsula meets the edge of the Antarctic polar vortex near Rothera due to a localized region of short horizontal wavelength gravity waves. This present study provides an extensive, climatological characterization of the mesospheric gravity-wave fields at two distinct stations that show not only the seasonal and day-today variation of gravity-wave momentum flux in the critical Antarctic region, but also the regional variations in that flux indicative of differences in wave forcing on the scale of the station separation, $1658 \mathrm{~km}$.

\section{Instrumentation and observations}

The sodium (Na) airglow, which originates from a thin ( $\sim 8 \mathrm{~km}$ thick) layer centred near $90 \mathrm{~km}$, is modulated as atmospheric wave-induced density and temperature perturbations move through the layer (Swenson and Gardner, 1998). As part of a collaboration between Utah State University (USU) and the British Antarctic Survey (BAS), observations of the gravity-wave induced intensity fluctuations of Na night airglow in two-dimensions were made using a monochromatic imaging system developed by USU. The camera used in this investigation, which has been described in detail elsewhere (Espy et al., 2004), consisted of a cooled $\left(-45^{\circ} \mathrm{C}\right)$, bare, $512 \times 512$ (effective) charge coupled device (CCD) array. This was coupled to a fast, $f / 4$, telecentric lens system to produce an all-sky image. Although a filter wheel was used to observe several airglow emissions, only the $\mathrm{Na}$ was used to calculate the momentum flux as it was the least susceptible to auroral contamination. A $2.5 \mathrm{~nm}$ band-pass filter

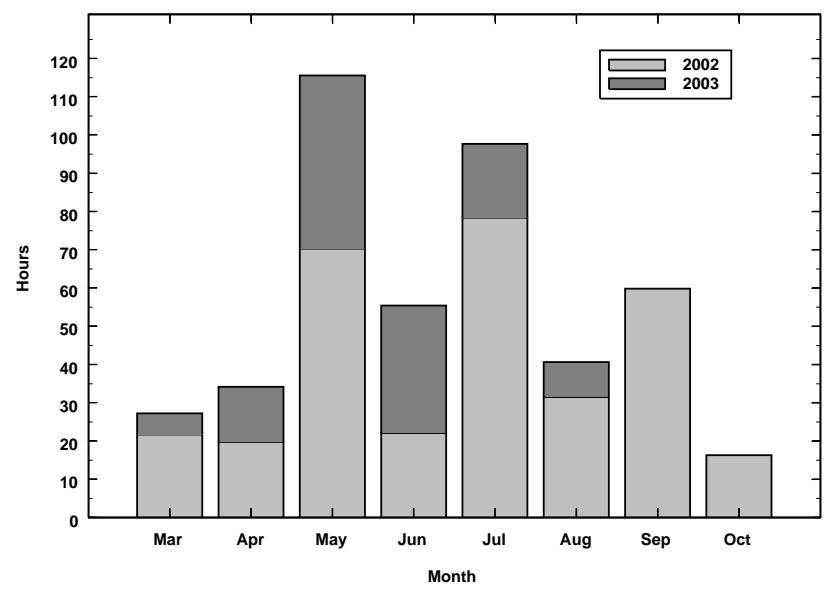

Fig. 2. The annual sampling distribution of usable image observations, in hours, at Rothera. The total number of hours in each month over the two years is given by the height of the bar, and the colour denotes the fraction of that total for the year 2002 (light grey) and 2003 (dark grey).

centred at $589 \mathrm{~nm}$ was used to isolate the $\mathrm{Na}$ emission. Each image was then integrated on the CCD for $90 \mathrm{~s}$ before being digitized to 16 bits, and the images were acquired at $\sim 6 \mathrm{~min}$ intervals.

Sodium images could be acquired whenever the sun had set $14^{\circ}$ or more below the horizon. In addition, the imager could tolerate a partial moon ( $\sim 40 \%$ disk) at elevations as high as $5^{\circ}$ before the CCD saturation spread and contaminated other pixels. Automated software was used so that images were acquired only under these conditions. With the high sensitivity of the camera system, gravity-wave perturbations of the $\mathrm{Na}$ airglow intensity of a few percent were readily observed. Waves observed by the imager are characterized by vertical wavelengths greater than $12 \mathrm{~km}$, short horizontal wavelengths $(\lambda<150 \mathrm{~km})$ and large phase speeds $\left(C_{I}>40 \mathrm{~m} / \mathrm{s}\right)$ (Swenson and Gardner, 1998). An example allsky Na image is shown in Fig. 1, where the north-to-south oriented gravity-wave structure is apparent.

Nightly sequences of these Na images were used to calculate the normalized momentum flux using the technique of Gardner et al. (1999), which is described in the next section. To estimate the daily averaged upper-limit of the vertical flux of horizontal momentum due to gravity waves, these normalized values were multiplied by the RMS wind perturbation due to the gravity waves. At Rothera, this was determined using coincident MF radar wind measurements and calculating the velocity variance over the same period to estimate the gravity-wave wind fluctuations (Meek et al., 1985).

The Rothera MF radar (Jarvis et al., 1999) is a coherent spaced-sensor radar system used for measuring horizontal winds in the mesosphere and lower thermosphere through the observation and analysis of D-region partial reflection echoes. The radar employs a single broad-beam transmit antenna that illuminates an area commensurate with the allsky imager field-of-view used in the analysis (Greet et al., 
2000), and three spaced receive antennas in a triangular array. The radar operates at a frequency of $1.98 \mathrm{MHz}$, with a peak transmitter power of $25 \mathrm{~kW}$ and full width half maximum pulse width of $25 \mu \mathrm{s}$. This corresponds to a height resolution of $\sim 4 \mathrm{~km}$ sampled at $2-\mathrm{km}$ height intervals between 50 and $100 \mathrm{~km}$, with the majority of echoes returned from altitudes between 75 and $95 \mathrm{~km}$. The received echoes are analyzed using the full-correlation analysis technique (Briggs, 1984) from which profiles of the zonal and meridional components of the horizontal wind are derived every two minutes (Vincent and Lesicar, 1991; Holdsworth et al., 1995).

The optical and radar observations were carried out during the austral winter seasons of 2002 and 2003 at Rothera for comparison with the results from the winter seasons of 2000 and 2001 at Halley (Espy et al., 2004). Despite the high geographic latitude of the stations, they are situated at lower geomagnetic latitude ( $\mathrm{L}=2.7$ and 4.5 for Rothera and Halley, respectively) than their northern latitude counterparts. Nevertheless, aurora could occasionally severely contaminate the images. Thus, the data for each night were screened for both auroral and meteorological cloud contamination using other, broadband spectral channels. For each night, a single contiguous image sequence that was free of aurora and cloud was selected for further analysis. The sequence length varied throughout the season from a maximum near solstice of $14.4 \mathrm{~h}$ to a minimum of $1.2 \mathrm{~h}$ near the equinoxes. In total, 49 days in 2002 and 28 days in 2003 were analysed at Rothera, in comparison to the 40 days in 2000 and 39 days in 2001 that had been used for Halley. Figure 2 gives the annual sampling distribution of usable imager observations in hours from Rothera. The MF-radar derived RMS wind at $90 \mathrm{~km}$, the nominal peak of the nighttime $\mathrm{Na}$ emission, was derived throughout the portion of the night during which airglow observations were available. The radar winds were available for all but 6 nights during 2002 at Rothera, in comparison to 4 missing nights of wind data in each of the two seasons at Halley.

\section{Analysis}

In this study, the measured azimuthal distribution of the relative airglow intensity variance is used as a proxy for the azimuthal distribution of the horizontal velocity variance in a gravity-wave model to predict the momentum flux as a function of azimuth angle. The technique for estimating momentum fluxes using airglow imager data in this way has been described fully by Gardner et al. (1999), and can be used to estimate the momentum fluxes associated with the full spectrum of both monochromatic as well as quasi-random wave perturbations. The technique has been validated against $\mathrm{Na}$ wind lidar measurements of momentum flux when applied to both monochromatic waves (Swenson et al., 1999) and the full wave spectrum (Gardner et al., 1999), and has been used to infer the seasonal variation of the momentum flux in the Northern and Southern Hemispheres (Tang et al. 2002; Espy et al., 2004)
Briefly, the 3-D Fourier spectrum in frequency, $\omega$, and the zonal, $k$, and meridional, $l$, horizontal wave numbers, was computed for the nightly sequence of all-sky $\mathrm{Na}$ images using the processing techniques detailed in Gardner et al. (1996) and Coble et al. (1998). The unambiguous 2-D horizontal wavenumber spectrum, describing the distribution of gravity-wave energy as a function of horizontal scale and propagation direction, was computed by integrating over frequency. This included the effects of waves with observed periods between $12 \mathrm{~min}$ (the temporal Nyquist limit) and $2 \mathrm{~h}$, and horizontal wavelengths between $2.4 \mathrm{~km}$ (the spatial Nyquist limit) and $150 \mathrm{~km}$. The unambiguous 2-D spectrum was then integrated over this wavenumber range to create an angular spectrum representing the azimuthal distribution of relative $\mathrm{Na}$ intensity variance.

Gardner et. al. (1999) has shown that the cross-correlation coefficients between the vertical and horizontal winds, which are the momentum fluxes normalized by the respective RMS wind perturbations, can be expressed as a simple integral over this azimuthal distribution of intensity variance as:

$$
\begin{gathered}
\frac{\left\langle w^{\prime}\left(\begin{array}{c}
u^{\prime} \\
v^{\prime}
\end{array}\right)\right\rangle}{w_{r m s}^{\prime}\left(\begin{array}{c}
u_{r m s}^{\prime} \\
v_{r m s}^{\prime}
\end{array}\right)}= \\
\frac{G \cdot \int_{0}^{2 \pi} d \phi \frac{\left\langle I_{N a}^{\prime}(\phi)^{2}\right\rangle}{\bar{I}_{N a}^{2}}\left(\begin{array}{c}
\sin (\phi) \\
\cos (\phi)
\end{array}\right)}{\left[\int_{0}^{2 \pi} d \phi \frac{\left\langle I_{N a}^{\prime}(\phi)^{2}\right\rangle}{\bar{I}_{N a}^{2}} \cdot \int_{0}^{2 \pi} d \phi \frac{\left\langle I_{N a}^{\prime}(\phi)^{2}\right\rangle}{\bar{I}_{N a}^{2}}\left(\begin{array}{c}
\sin ^{2}(\phi) \\
\cos ^{2}(\phi)
\end{array}\right)\right]},
\end{gathered}
$$

where $w_{r m s}^{\prime}, u_{r m s}^{\prime}$ and $v_{r m s}^{\prime}$ are the RMS values of vertical and horizontal zonal and meridional wind perturbations, while $\bar{I}_{N a}$ and $I_{N a}^{\prime}$ are the mean value and relative perturbation of the $\mathrm{Na}$ emission intensity caused by gravity waves moving in the azimuthal direction, $\phi$. The vertical flux of horizontal momentum is directly proportional to these correlation coefficients scaled by the standard deviations of the horizontal and vertical winds derived from the radar data.

The above relations and the factor, $G$, which sets the range of the cross-correlations coefficients, were developed using the gravity wave polarization relations and employing a canonical power-law model for the frequency distribution of horizontal velocity variance. As a model frequency spectrum ( spectral slope $=2$ ) is integrated from the inertial to the Brunt frequency, it is not necessary to compensate for the Doppler effects associated with the mean background wind. For the exact form of $G$ and uncertainties of the zonal and meridional cross-correlation coefficients, the reader is referred to Gardner et al. (1999). As the method assumes that all waves propagate upwards and are not ducted, it provides an upper limit to the momentum flux (Fritts, 2000). However, this does not appear to be a serious shortcoming. By adjusting the integration limits, Tang et al. (2002) showed that the ducted waves, typically with horizontal wavelengths less than $20 \mathrm{~km}$ (Fritts and Alexander, 2003), contribute less than $10 \%$ to the momentum fluxes inferred using this method, 

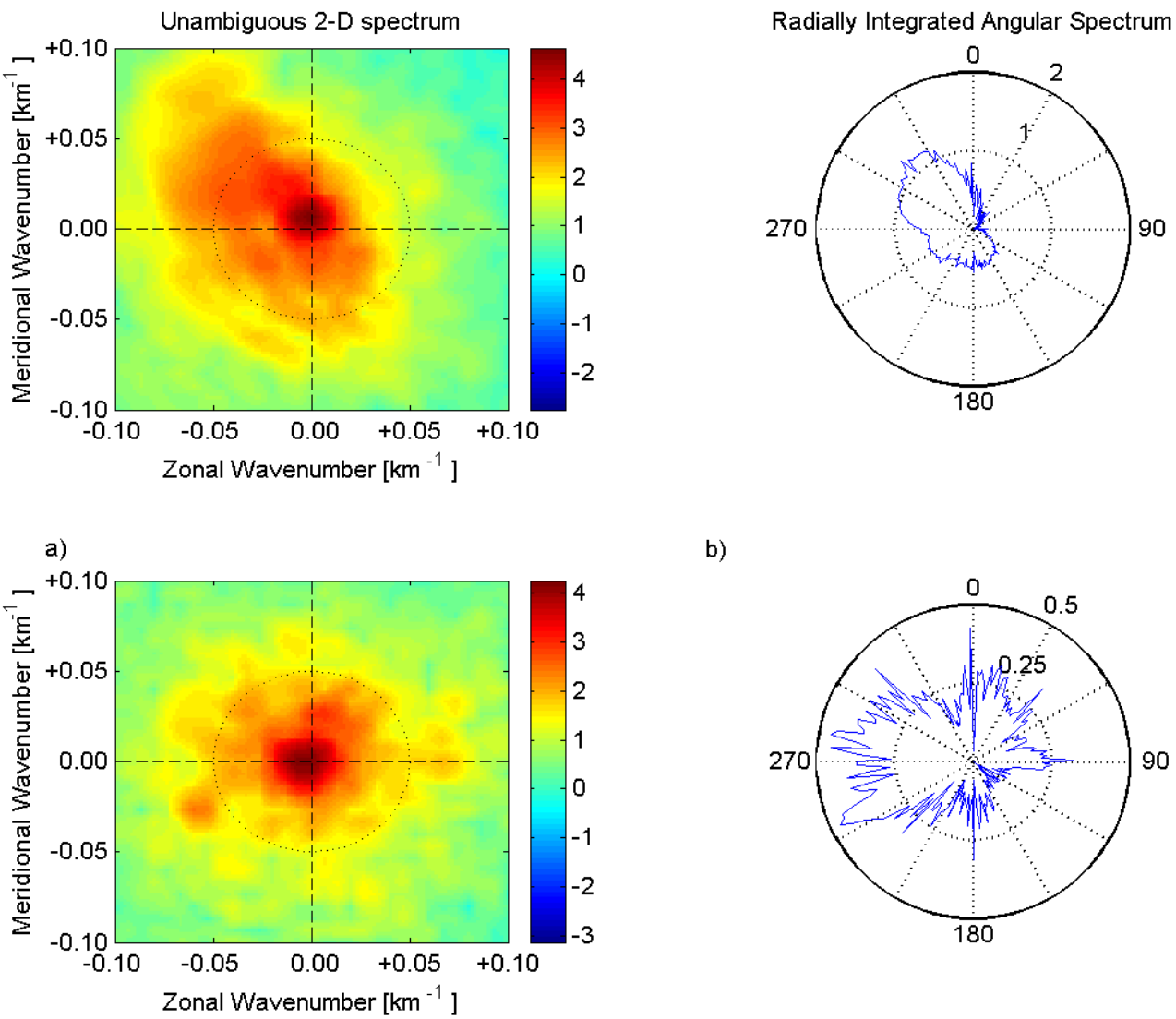

b)

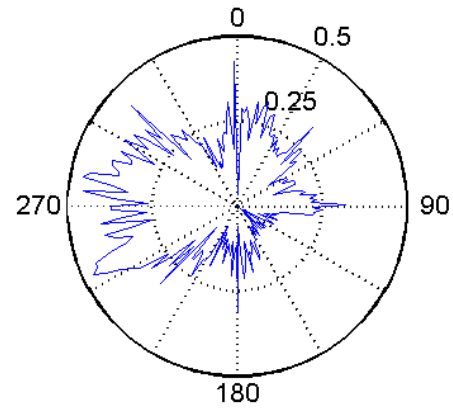

c)

d)

Fig. 3. The unambiguous 2-D horizontal wavenumber spectrum, (a), and the angular spectrum, (b), for the sequence of images acquired during the night of 28/29 July 2002 from Rothera. The scales for both spectra are logarithmic, and the 2-D spectrum is in units of (relative $\%$ wave amplitude/ wavenumber) ${ }^{2}$, whilst the angular spectrum has units of (relative $\%$ wave amplitude $)^{2} /$ radian. For comparison, the wavenumber spectrum, (c), and the angular spectrum, (d), for the same night during 2001 at Halley are shown.

and those results have been confirmed in the present analysis. In addition, Gardner et. al. (1999) successfully validated the momentum fluxes derived using this imager-spectra technique against those measured directly by a dual-beam $\mathrm{Na}$ wind lidar. Due to the extended gravity-wave spectrum observed, Swenson et al. (1999) found that the magnitudes of the imager-spectra and Na-lidar momentum fluxes reported by Gardner et al. (1999) were larger than those derived from imaging specific monochromatic waves or the climatological values derived from radars (Vincent, 1984). Thus, only momentum-flux magnitudes derived using this same imagerspectra technique described by Gardner et. al. (1999) will be compared here.

To employ this procedure, the true zenith pixel and northward direction were determined using star positions. The images were then re-centred on this position and rotated so as to align the cardinal directions along the Cartesian axis. Next, the images were flat-fielded to remove the van Rhijn effect and to correct for the variations in the response of the fish-eye lens and CCD (Coble et al., 1998). Finally, the stellar contamination was removed using the gradient- based edge technique described by Tang et al. (2003). To avoid the severe distortions and loss of spatial resolution introduced by the fish-eye lens, only the centre 150-by- $150 \mathrm{~km}$ field-of-view was interpolated to a 256 square grid on geographic co-ordinates. The unambiguous 2-D Fourier spectrum of the airglow perturbations was then calculated for each night using the sequence of these re-sampled images (Gardner et al., 1996; Coble et al., 1998; Tang et al., 2002). The angular spectrum, which gives the distribution of wave energy as a function of propagation direction, was calculated for each night by integrating the resulting unambiguous Fourier spectrum radially over horizontal wave numbers from $2 \pi /(150 \mathrm{~km})$ to $2 \pi /(2.4 \mathrm{~km})$, the maximum field and spatial Nyquist limit, respectively.

As an example, the unambiguous 2-D horizontal wavenumber spectrum and the angular spectrum for the night of 28/29 July 2002 from Rothera is shown in Figs. 3a and b. For comparison, the same day in 2001 from Halley is shown in Figs. 3c and d. The 2-D spectra show extensive wave amplitudes at both Rothera and Halley. However, the gravity wave amplitudes at Rothera are predominantly in the 


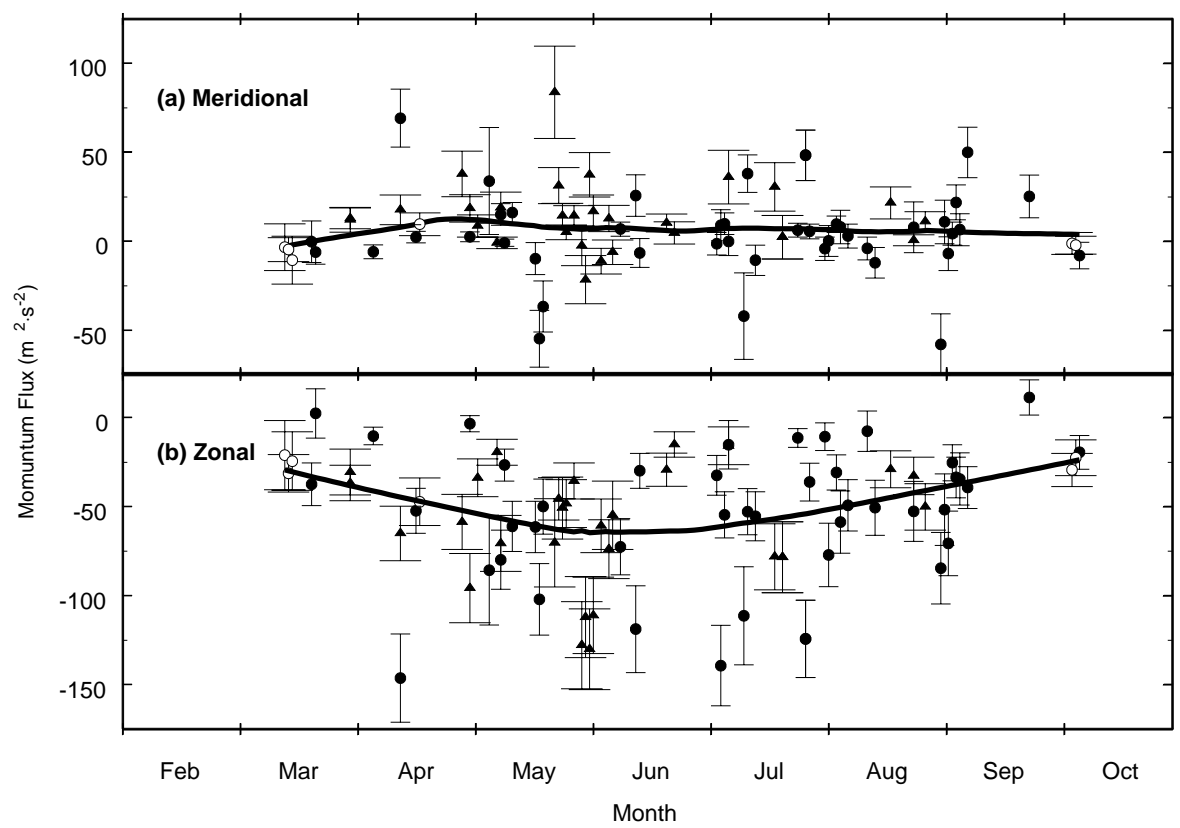

Fig. 4. The daily meridional (a) and zonal (b) momentum fluxes for the years 2002 and 2003 at Rothera. The data for 2002 are represented by circles and those for 2003 are given by the triangles. Data for which there are no coincident MF-radar winds are shown by the open symbols, and the solid lines are a weighted least-squares fit to the data.

westward (negative wavenumber) direction as opposed to the more uniform distribution at Halley. The direction of the wave field is more clearly indicated by the angular spectra of Figs. 3b and d. Qualitatively, more gravity-wave amplitude is seen in the westward propagating direction than the eastward at Rothera, which suggests a large zonal momentum flux there. Also, the gravity-wave amplitudes at Halley are more nearly balanced in the eastward and westward directions, implying a smaller net zonal momentum flux there.

Using this angular spectrum, the relative Na intensity variance was then determined as a function of azimuth angle for use as a proxy for the horizontal velocity variance in the expressions for the cross-correlation coefficients between the horizontal and vertical winds (Coble et al., 1998; Gardner et. al., 1999). The radar winds over the $\mathrm{Na}$ airglow layer altitude, $90 \mathrm{~km}$, were binned into half-hourly medians, and the mean and tidal components fitted over sliding 3-day intervals were removed (Charles and Jones, 1999). The variance of the residuals was calculated over the same time period as the imager data so as to capture the same frequency content, and this was used to represent the variance due to gravity waves with periods between $4 \mathrm{~min}$ (the Nyquist period) and the length of the data segment (Meek et al., 1985). Averaging three adjacent range bins to approximate the response of the thicker Na layer did not significantly affect the variance determination, but reduced the number of points available for the tidal fit. As the effects were negligible, and in order to improve the quality of the tidal fits and more closely duplicate the Halley analysis, only the $90 \mathrm{~km}$ range bin was used to determine the wind variance. To estimate the errors associated with these wind variances, this procedure was performed to calculate daily variance values throughout the years of operation at each station. The variance levels were at their lowest during the summer periods due to lower gravity-wave fluxes (Lübken et al., 1990), and the absolute minimum daily value was taken to be a measure of the uncertainty in the variance for use in the error analysis. Although this estimate of uncertainty may contain residual geophysical variations, it represents an upper limit of the uncertainty in the calculated wind variance. These wind variances were then used to scale the correlation coefficients so as to determine the nightly averaged vertical flux of horizontal momentum in the zonal and meridional directions, and the error estimates for the variances and correlation coefficients propagated through the calculation to provide an uncertainty estimate for the momentum flux. For the nights where radar winds were not available, the average yearly values of the wind variances were used as scaling factors (Tang et al., 2002; Espy et al., 2004).

\section{Results}

The results for the two seasons at Rothera are shown in Fig. 4. Here we see that the momentum fluxes in both zonal and meridional directions show a great deal of day-to-day variability, a feature noted in other seasonal studies, particularly at Halley (Murphy and Vincent, 1993; Swenson et. al., 1999; Tang et al., 2002; Espy et al., 2004). However, despite the variability, there is a tendency for the large westward (negative) zonal values to occur near winter solstice and to approach zero near the equinoxes. Those points for which 


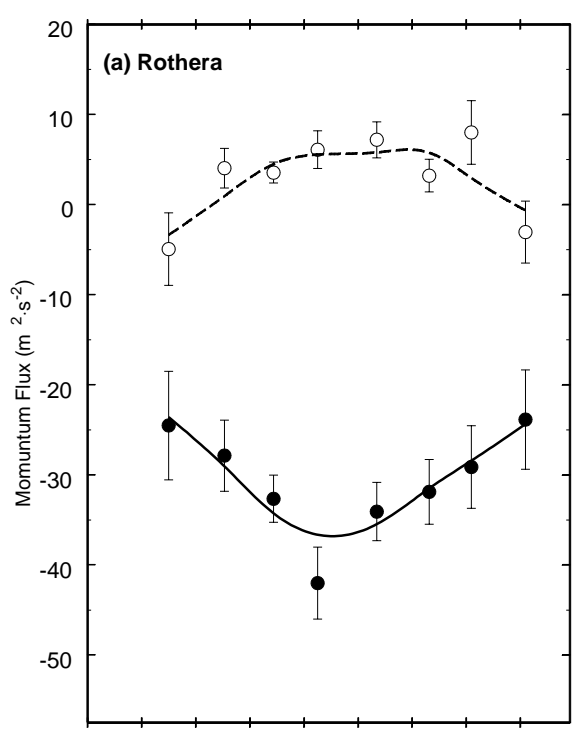

Feb Mar Apr May Jun Jul Aug Sep Oct

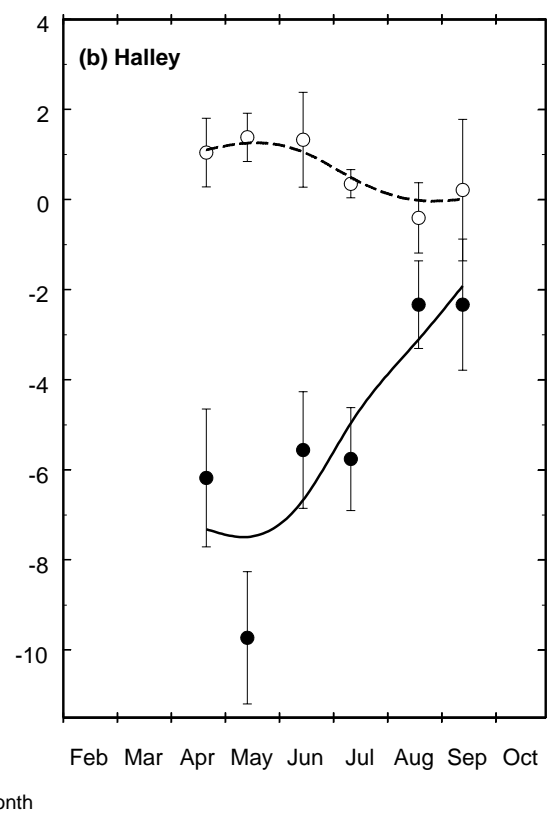

Month

Fig. 5. The monthly average zonal (solid circles) and meridional (open circles) momentum fluxes from Rothera (a). Also shown in (b) are the monthly averages from Halley, on a vertical scale 5 times smaller than the Rothera results. The solid and dashed lines are weighted least-squares fits to the zonal and meridional data, respectively.

the average values of the wind variances were used as scaling factors, shown by the open data points, do not show a significant trend, indicating that this approximation does not bias the results. Also, the zonal fluxes appear to be generally in the westward direction and much stronger than the northward (positive) tending meridional fluxes.

The individual nightly gravity-wave momentum-flux measurements from the two years at Rothera were averaged to monthly means to examine the climatological seasonal behaviour. The uncertainties in each nightly value were used to weight the values in the monthly average, which are shown along with their standard errors of the mean in Fig. 5a. For comparison, the Halley monthly averages from Espy et al. (2004), obtained using the same analysis technique, are reproduced in Fig. 5b. The momentum fluxes from both stations show a clear trend to shift from the north and strongly westward at mid-winter towards the south and weakly westward near the equinoxes, consistent with the filtering of gravity waves by the underlying wind field (Espy et al., 2004). This behaviour is in agreement with other southern-hemisphere seasonal observations (Murphy and Vincent, 1993) and with northern-hemisphere results, although there the meridional trends are of course reversed (Swenson et. al., 1999; Tang et al., 2002). Whilst both Rothera and Halley display wintertime westward and northward flux, the magnitude of the monthly average flux at Rothera is $\sim 4$ times greater. The trend in both the seasonal variation and the difference between the Rothera and Halley momentum fluxes are observed in the raw correlation coefficients derived from the imager, which is common to the two sites. These variations and differences are then amplified when the correlation coefficients are scaled by the wind variances to obtain the momentum flux. The two different radar techniques used to obtain these wind variances, MF at Rothera and IDI at Halley, have each been found to compare favourably with meteor wind radars (Hocking and Thayaparan, 1997; Thayaparan and Hocking, 2002; Jones et al., 2003), indicating that the larger momentum fluxes at Rothera are not the result of the different radar techniques. In fact, the tendency for MF radars to underestimate the winds above $90 \mathrm{~km}$ (e.g. Hocking and Thayaparan, 1997; Thayaparan and Hocking, 2002) would further increase the Rothera momentum fluxes.

Averaging the individual daily values shown in Fig. 4 yields a mean flux at Rothera of $-32 \mathrm{~m}^{2} \cdot \mathrm{s}^{-2}$ and $4.0 \mathrm{~m}^{2} \cdot \mathrm{s}^{-2}$ in the zonal and meridional directions, respectively. As with the monthly averages, this is significantly higher than the wintertime average flux observed using the same technique at Halley of $-7.5 \mathrm{~m}^{2} \cdot \mathrm{s}^{-2}$ in the zonal and $1.6 \mathrm{~m}^{2} \mathrm{~s}^{-2}$ in the meridional directions (Espy et al., 2004). However, the zonal difference is much smaller when the Rothera flux is compared to that observed at a similar mountainous site at $35^{\circ} \mathrm{N}$ using the same technique. There, Tang et al. (2002) observed an average wintertime flux at $35^{\circ} \mathrm{N}$ of $-20 \mathrm{~m}^{2} \cdot \mathrm{s}^{-2}$ and $-12 \mathrm{~m}^{2} \cdot \mathrm{s}^{-2}$ in the zonal and meridional directions, respectively.

One possibility is that higher stratospheric winds may produce more efficient filtering of the eastward gravity waves at the different sites, thus creating more asymmetry in the wave field and correspondingly greater westward momentum flux reaching the mesosphere. However, the Horizontal Wind Model (HWM-93) (Hedin et al., 1996) shows a 
wintertime stratospheric zonal-wind maximum at Rothera of $\sim 53 \mathrm{~m} \cdot \mathrm{s}^{-1}$, as compared to $\sim 57 \mathrm{~m} \cdot \mathrm{s}^{-1}$ at $35^{\circ} \mathrm{N}$ and $\sim 40 \mathrm{~m} \cdot \mathrm{s}^{-1}$ at Halley. Thus, while decreased efficiency of the stratospheric wind filtering may play some role in the low zonal flux at Halley, it cannot explain the zonal flux at Rothera being $50 \%$ larger than that at $35^{\circ} \mathrm{N}$. Similarly, the HWM-93 stratospheric meridional winds, which are similar at the three sites, cannot explain the differences observed in the meridional-flux.

A more likely explanation is that the gravity-wave source functions themselves differ at the three sites. While similar at the mountainous sites, Halley's flat topography, as well as its distance from the Trans-Antarctic Mountains, the storm systems of the South Atlantic and the shear zones of the wintertime polar vortex, all contribute to a smaller gravity-wave source function there. This would account for the significant regional differences in the momentum flux between Rothera and Halley, and hence the wave forcing, on the scale of the station separation.

\section{Conclusions}

Short-scale gravity waves play an essential role determining the global circulation and thermal balance. GCM parameterizations of these waves must be both guided and constrained by measurements of critical parameters, such as the gravity-wave momentum flux, its seasonal change and its regional variation, particularly in the critical Antarctic regions. To that end, we have used 77 nights, distributed over two years, of Antarctic Na-airglow imaging observations and coincident MF-radar winds from Rothera to derive the momentum flux using the 3-D spectral analysis technique of Gardner et. al. (1999). The resulting seasonal momentum-flux climatology may then be directly compared to that reported from Halley using a similar technique (Espy et al., 2004) to examine regional variations indicative of differences in wave activity on the scale of the station separation, $1658 \mathrm{~km}$.

The results from Rothera show a great deal of day-to-day variability in the derived momentum flux, but a distinct tendency for the mid-winter, strong westward momentum flux to approach zero near the equinoxes. Combining the data into monthly averages, the seasonal variation showing the turning of the momentum flux from the north and strongly westward direction at mid-winter towards the south and weakly westward direction at the equinoxes is seen clearly. This seasonal trend has been shown to be consistent with the stratospheric winds preferentially removing gravity waves with their same directions through critical-level filtering (Alexander, 1998; Fritts and Alexander, 2003; Espy et al., 2004). Thus, the remaining gravity-wave field in the mesosphere is observed to rotate so as to track the summer pole (Tang et al., 2002).

Although this rotation of the momentum flux is observed at both Rothera and Halley, the magnitude of the Rothera zonal flux climatology is $\sim 4$ times greater than that observed at Halley. This is in agreement with the results of Ern et al. (2004), who observed a similar spatial maximum of gravity-wave momentum flux near Rothera during a twoweek period. Similarly, the zonal fluxes at Rothera are $\sim 50 \%$ larger than those observed using the same technique at a northern mid-latitude site with similar orographic forcing. These flux differences cannot be explained by variations in stratospheric wind filtering alone, indicating that the gravitywave sources are systematically stronger at Rothera.

In summary, the observational results presented here show that the seasonal variation of the horizontal momentum carried upward through the $\mathrm{Na}$ airglow region by highfrequency gravity waves is directed opposite to the stratospheric wind direction. In both Antarctica and the northern hemisphere, this results in wintertime fluxes that are predominantly zonal while the meridional fluxes display a marked rotation towards the summer pole throughout the season. In addition, Rothera, which is located on the mountainous Antarctic Peninsula, must also have stronger gravitywave sources to account for its flux being approximately four times greater than that observed at Halley on the Brunt Ice Shelf. Thus, differences over $\sim 1700 \mathrm{~km}$ in both the gravitywave source functions and possibly filtering by the underlying winds can create significant regional differences in momentum flux and consequent wave forcing over Antarctica throughout the autumn through spring seasons.

Acknowledgements. The Halley imager was jointly supported by US National Science Foundation grants ATM-0003180 and OPP9816465, and by the UK Natural Environment Research Council. Funding for D. Fritts and D. Riggin was provided by the National Science Foundation through grant ATM-0438777.

Topical Editor U.-P. Hoppe thanks N. J. Mitchell and another referee for their help in evaluating this paper.

\section{References}

Alexander, M. J.: Interpretations of observed climatological patterns in stratospheric gravity wave variance, J. Geophys. Res., 103, 8627-8640, 1998.

Briggs, B. H.: The analysis of spaced sensor records by correlation techniques, in: Handbook for MAP, SCOSTEP Secr., Univ. of Ill., Urbana, vol. 13, 166-186, 1984.

Charles, K. and Jones, G. O. L.: Mesospheric mean winds and tides observed by the imaging Doppler interferometer (IDI) at Halley, Antarctica, J. Atmos. Sol. Terr. Phys., 61, 351-362, 1999.

Coble, M. R., Papen, G. C. and Gardner, C. S.: Computing twodimensional unambiguous horizontal wavenumber spectra from OH airglow images, IEEE Trans. Geosci. and Remote Sens., 36, 368-382, 1998.

Ern, M., Preusse, P., Alexander, M. J., and Warner, C. D.: Absolute values of gravity wave momentum flux derived from satellite data, J. Geophys. Res., 109, D20103, doi:10.1029/2004JD004752, 2004.

Espy, P. J. and Stegman, J.: Trends and variability of mesospheric temperature at high-latitudes, Physics and Chemistry of the Earth, 27, 543-553, 2002.

Espy, P. J., Jones, G. O. L., Swenson, G. R., Tang, J., and Taylor, M. J.: Seasonal variations of the gravity-wave momentum flux in the Antarctic mesosphere and lower thermosphere, J. Geophys. Res., 109, D23109, doi:10.1029/2003JD004446, 2004. 
Fritts, D. C.: Errant influences of gravity wave momentum and heat fluxes using airglow and lidar instrumentation: Corrections and cautions, J. Geophys. Res., 105, 22 355-22 360, 2000.

Fritts, D. C. and Luo, Z. G.: Dynamical and radiative forcing of the summer mesopause circulation and thermal structure, 1. Mean solstice conditions, J. Geophys. Res., 100, 3119-3128, 1995.

Fritts, D. C. and Alexander, M. J.: Gravity wave dynamics and effects in the middle atmosphere, Rev. Geophys., 41(1), 1003, doi:10.1029/2001RG000106, 2003.

Garcia, R. R. and Solomon, S.: The effect of breaking gravity waves on the dynamics and chemical composition of the mesosphere and lower thermosphere, J. Geophys. Res., 90, 3850-3868, 1985.

Garcia, R. R. and Boville, B. A.: "Downward control" of the mean meridional circulation and temperature distribution of the polar winter stratosphere, J. Atmos. Sci., 51, 2238-2245, 1994.

Gardner, C. S., Coble, M., Papen, G. C., and Swenson, G. R.: Observations of the unambiguous 2-dimensional horizontal wave number spectrum of $\mathrm{OH}$ intensity perturbations, Geophys. Res. Lett, 23, 3739-3742, 1996.

Gardner, C. S., Gulati, K., Zhao, Y., and Swenson, G.: Measuring gravity wave momentum fluxes with airglow imagers, J. Geophys. Res., 104, 11 903-11915, 1999.

Greet, P. A., Murphy, D. J., Vincent, R., and Dyson, P. L.: A comparison of optical and radar measurements of mesospheric winds and tides, Geophys. Res. Lett, 27, 2477-2480, 2000.

Hamilton, K.: Comprehensive modeling of the middle atmosphere climate: Some recent results, Climate Dynamics, 11, 223-241, 1995.

Hamilton, K.: Comprehensive meteorological modeling of the middle atmosphere: A tutorial review, J. Atmos. Terr. Phys., 58, 1591-1627, 1996.

Haurwitz, B.: Frictional effects and the meridional circulation in the mesosphere, J. Geophys. Res., 66, 2381-2391, 1961.

Hedin, A. E., Fleming, E. L., Manson, A. H., Schmidlin, F. J., Avery, S. K., Clark, R. R., Franke, S. J., Fraser, G. J., Tsuda, T., Vial, F., and Vincent, R. A.: Empirical wind model for the upper, middle and lower atmosphere, J. Atmos. Terr. Phys., 58, 14211447, 1996.

Hocking, W. K. and Thayaparan, T.: Simultaneous and colocated observation of winds and tides by MF and meteor radars over London, Canada ( $\left.43^{\circ} \mathrm{N}, 81^{\circ} \mathrm{W}\right)$, during 1994-1996, Radio Sci., 32, 833-866, doi:10.1029/96RS03467, 1997.

Holdsworth, D. A. and Reid, I. M.: A simple model of atmospheric radar backscatter: Description and application to the full correlation analysis of spaced antenna data, Radio Sci., 30, 1263-1280, 1995.

Jarvis, M. J., Jones G. O. L., and Jenkins, B.: New initiatives in observing the Antarctic mesosphere, Adv. Space Res., 24, 611$619,1999$.
Jones G. O. L., Berkey, F. T., Fish, C. S., Hocking, W. K., and Taylor, M. J.: Validation of imaging Doppler interferometer winds using meteor radar, Geophys. Res. Lett., 30, 1743, doi:10.1029/2003GL017645, 2003.

Lübken, F.-J., von Zahn, U., Manson, A., Meek, C., Hoppe, U.-P., Schmidlin, F. J., Stegman, J., Murtagh, D. P., Rüster, R., Schmidt, G., Widdel, H.-U., and Espy, P.: Mean state densities, temperatures and winds during the MAC/SINE and MAC/EPSILON campaigns, J. Atmos. Terr. Phys., 52, 955-970, 1990.

Luo, Z. G., Fritts, D. C., Portmann, R. W., and Thomas, G. E.: Dynamical and radiative forcing of the summer mesopause circulation and thermal structure, 2. Seasonal-variations, J. Geophys. Res., 100, 3129-3137, 1995.

Meek, C. E., Reid, I. M., and Manson, A. H.: Observations of mesospheric wind velocities, 1 . Cross section of power spectral density for 48-8 hours, 8-1 hours, and 1 hour to 10 min over 60 $110 \mathrm{~km}$ for 1981, Radio Science, 20, 1383-1402, 1985.

Murphy, D. J. and Vincent, R. A.: Estimates of momentum flux in the mesosphere and lower thermosphere over Adelaide, Australia, from March 1985 to February 1986, J. Geophys. Res., 98, 18 617-18 638, 1993.

Rind, D., Suozzo, R., Balachandran, N. K., Lacis, A. and Russell, G.: The GISS global climate-middle atmosphere model, Part I: Model structure and climatology, J. Atmos. Sci., 45, 329-370, 1988.

Swenson, G. R. and Gardner, C. S.: Analytical models for the responses of the mesospheric $\mathrm{OH}^{*}$ and Na layers to atmospheric gravity waves, J. Geophys. Res., 103, 6271-6294, 1998.

Swenson, G. R., Haque, R., Yang, W., and Gardner, C. S.: Momentum and energy fluxes of monochromatic gravity waves observed by an $\mathrm{OH}$ imager at Starfire Optical Range, New Mexico, J. Geophys. Res., 104, 6067-6080, 1999.

Tang, J., Liu, A. Z., and Swenson, G. R.: Height frequency gravity waves observed in $\mathrm{OH}$ airglow at Starfire Optical Range, NM: Seasonal variations in momentum flux, Geophys. Res. Lett., 29, 1966-1969, 2002.

Tang, J., Kamalabadi, F., Rumsey, L. G., and Swenson, G. R.: Point source suppression for atmospheric wave extraction from airglow imaging measurements, IEEE Trans. Geosci. and Remote Sens., 41, 146-152, 2003.

Thayaparan, T. and Hocking, W. K.: A long-term comparison of winds and tides measured at London, Canada $\left(43^{\circ} \mathrm{N}, 81^{\circ} \mathrm{W}\right)$ by co-located MF and meteor radars during 1994-1999, J. Atmos. Solar-Terr. Phy., 64, 931-946, 2002.

Vincent, R. A.: Gravity-wave motions in the mesosphere, J. Atmos. Terr. Phys., 46, 119-128, 1984.

Vincent, R. A. and Lesicar, D.: Dynamics of the equatorial mesosphere: First results with a new generation partial reflection radar, Geophys. Res. Lett., 18, 825-828, 1991. 